Between September 2000 and April 2006, 125 patients underwent PPVI at the study center. From this population, the researchers identified 17 patients who had a pulmonary regurgitation without major obstruction of the right ventricular outflow tract. All patients underwent cardiopulmonary exercise testing, tissue Doppler echocardiography and MRI before and approximately 1 month after PPVI. NYHA heart failure class improved from a median of 2 before surgery to a median of 1 after the procedure $(P=0.001)$. Following surgery, pulmonary regurgitant fraction fell significantly (from a mean of $40.7 \%$ to a mean of $4.1 \% ; P<0.001)$, as did right ventricular enddiastolic volume (from a mean of $116.7 \mathrm{ml} / \mathrm{m}^{2}$ to a mean of $100.2 \mathrm{ml} / \mathrm{m}^{2} ; P=0.001$ ). Improvements in effective stroke volume, left ventricular stroke volume, left ventricular ejection fraction and cardiac output were also reported. By contrast, maximal exercise tests completed by 15 of the patients after PPVI did not demonstrate any improvements in exercise capacity; peak $\mathrm{VO}_{2}$, anaerobic threshold and workload measurements were not significantly different from those made at baseline.

The authors conclude that, in contrast to that of the pressure-overloaded ventricle, the contractile reserve of the volume-overloaded ventricle is minimal.

Original article Coats L et al. (2007) Physiological consequences of percutaneous pulmonary valve implantation: the different behaviour of volume- and pressure-overloaded ventricles. Eur Heart J 28: 1886-1893

\section{Primary care practitioners struggle to accurately diagnose AF on electrocardiography}

A diagnosis of atrial fibrillation (AF) is often made in primary care facilities. Mant and colleagues investigated the ability of general practitioners (GPs), nurses, and interpretive software to make accurate diagnoses of AF on the basis of electrocardiograms. A total of 2,595 electrocardiograms, taken from participants in the SAFE trial, were randomly sent to 1 of 49 general practices in the UK for assessment. The resulting diagnoses were compared for accuracy against those made by two consultant cardiologists.

AF was present in $8.4 \%$ of the study population, all of whom were aged 65 years or over. Using 12-lead electrocardiograms, GPs failed to diagnose 20 out of 99 true cases (sensitivity $79.8 \%$ ), and made an incorrect diagnosis in 114 of the 1,355 patients without AF (specificity $91.6 \%$ ). Primary care nurses fared similarly, displaying a sensitivity of $77.1 \%$ and specificity of $85.1 \%$ using 12-lead traces. The interpretive software was more accurate than both GPs and nurses (sensitivity $83.3 \%$, specificity $99.1 \%$ ), but still missed 36 out of 215 true cases of AF, and misdiagnosed 21 out of the 2,341 patients who did not have the condition.

These findings indicate that electrocardiograms can often be misinterpreted by primary care practitioners, highlighting the need for greater quality control.

Original article Mant J et al. (2007) Accuracy of diagnosing atrial fibrillation on electrocardiogram by primary care practitioners and interpretative diagnostic software: analysis of data from screening for atrial fibrillation in the elderly (SAFE) trial. BMJ 335: 380

\section{Hospital treatment of heart failure improved by participation in OPTIMIZE-HF}

The quality of care given to patients with heart failure (HF) can be quantified according to evidence-based guideline recommendations. The OPTIMIZE-HF study set out to improve adherence to these performance measures.

Data were collected on 48,612 patients (mean age 73.1 years) hospitalized with HF at 259 hospitals in the US. Patient information was added to an online registry that enabled hospitals to monitor their own performance in real time, benchmarked against figures from other centers. The study also provided various tools to assist with the optimum management of HF.

Participation in OPTIMIZE-HF increased the proportion of eligible patients who received left ventricular function assessment (from 89\% to 92\%; $P<0.001)$, advice on smoking cessation (from $48 \%$ to $76 \% ; P<0.001$ ), and discharge instructions regarding diet, medications and weight (from $47 \%$ to $67 \%$; $P<0.001$ ). The prescription of $\beta$-blockers and aldosterone antagonists at discharge also improved, as did the appropriate use of statins and anticoagulation therapy. There was, however, no significant improvement in the use of angiotensin-converting-enzyme inhibitors or angiotensin-receptor blockers in eligible patients. 